\title{
Nail Unit Glomus Tumor with Myxoid and Symplastic Change Presenting with Longitudinal Erythronychia
}

\author{
Diego R. Da Silva ${ }^{a}$ Kevin J. Gaddis ${ }^{b}$ Stephen Hess ${ }^{c}$ Adam I. Rubin ${ }^{a}$ b \\ aPerelman School of Medicine at the University of Pennsylvania, Philadelphia, PA, USA; \\ ${ }^{b}$ Department of Dermatology, Hospital of the University of Pennsylvania, \\ Philadelphia, PA, USA; ${ }^{c}$ Center City Dermatology, Philadelphia, PA, USA
}

\section{Keywords}

Glomus tumor · Longitudinal erythronychia · Myxoid change · Symplastic change

\begin{abstract}
Glomus tumors are soft tissue neoplasms, which are most frequently encountered in the nail unit and generally straightforward to diagnose by histopathology. The typical clinical presentation is that of a circular violaceous or erythematous lesion within the nail bed. However, there are rare variants of glomus tumors which may pose diagnostic challenges because of the presence of unusual histologic features. Herein we report such a glomus tumor that demonstrates the rare combination of both myxoid and symplastic change. The clinical presentation of longitudinal erythronychia, as seen with this case, can occur with glomus tumors, but it is unusual, as longitudinal erythronychia on a single nail usually is caused by an onychopapilloma. The distinct nuclear atypia characteristic of symplastic change can raise alarm for a malignant process but the clinical course is benign. It is essential for dermatopathologists to be aware of this unusual variant of a glomus tumor in order to avoid overdiagnosis of atypia, which could result in unnecessary aggressive surgery. While unusual, there is good clinicopathologic correlation of the glomus tumor presenting with longitudinal erythronychia.
\end{abstract}


1

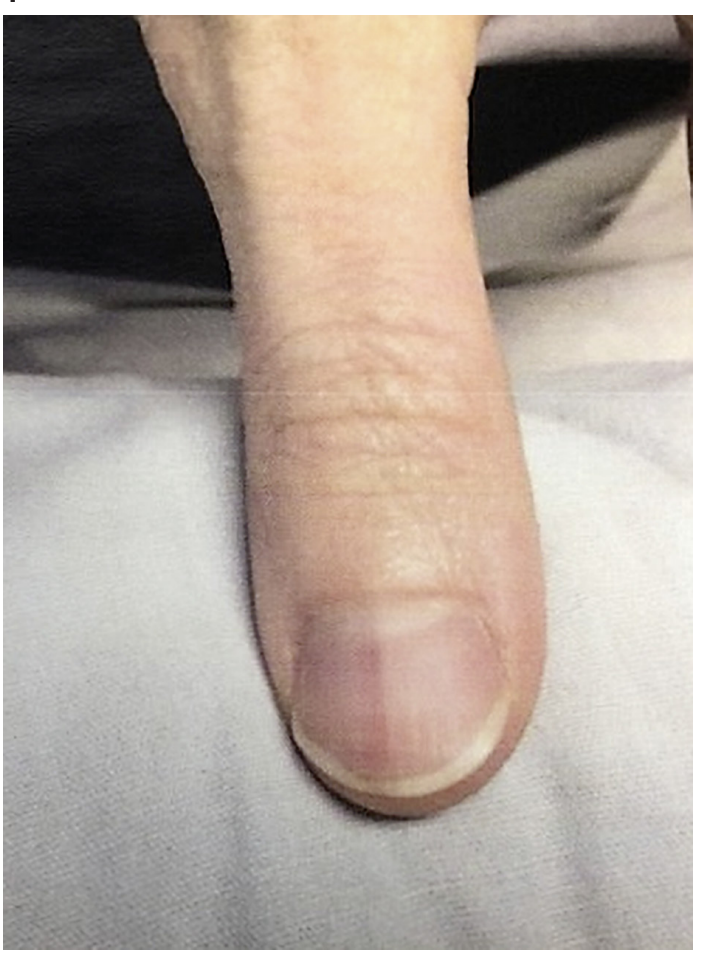

2

(C) 2018 The Author(s). Published by S. Karger AG, Basel www.karger.com/dpa

Da Silva et al.: Nail Unit Glomus Tumor with Myxoid and Symplastic Change

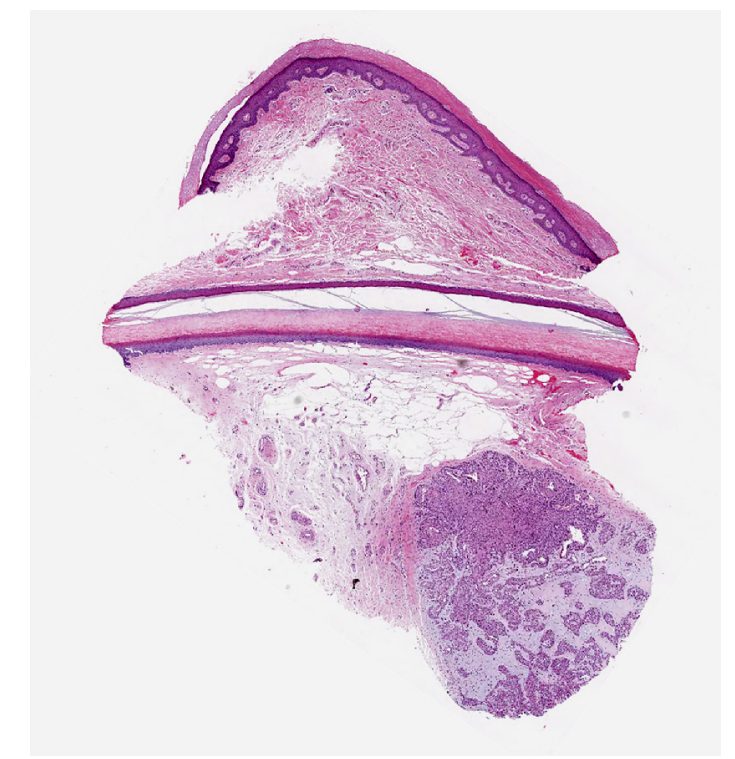

Fig. 1. The left thumbnail shows a thin band of longitudinal erythronychia that extends from the cuticle to the hyponychium.

Fig. 2. Punch biopsy of the nail unit that demonstrates anatomic areas of the dorsal and ventral aspects of the proximal nail fold, nail plate, matrix epithelium, and nail unit connective tissue. At the deep aspect of the specimen, a tumor with vascular channels and myxoid change is seen (hematoxylin and eosin, $\times 20$ ).

\section{Case Report}

A 41-year-old female presented to her dermatologist's office for evaluation of the left thumbnail. There was a 10-year history of longitudinal erythronychia of the left thumbnail, and recently there was the onset of associated pain of the nail with palpation and pressure. The patient denied a history of trauma or infection in the affected nail. An X-ray of the nail did not demonstrate underlying bone or soft tissue pathology. Over the next few months, the lesion progressed, with deepening of the erythronychia, formation of a longitudinal ridge in the nail plate, and progressive increase in pain intensity (Fig. 1). A biopsy of the left thumbnail unit was performed.

\section{Histopathology}

Sections of this nail unit specimen revealed a deep dermal nodule lying beneath the associated matrix epithelium which was comprised of eosinophilic, cuboidal cells forming vascular luminae. Foci of myxoid stromal changes were present. Scattered enlarged, atypical cells with hyperchromatic and irregularly contoured nuclei were present. However, extensive examination of the lesional cells did not demonstrate mitoses. Focally prominent nucleoli were also present in these cells. Immunohistochemical analysis demonstrated the lesional cells to be highlighted with smooth muscle actin. A PHH3 stain did not highlight the lesional cells. These features were diagnostic of a nail unit glomus tumor with myxoid and symplastic change (Fig. 2-7). 
3

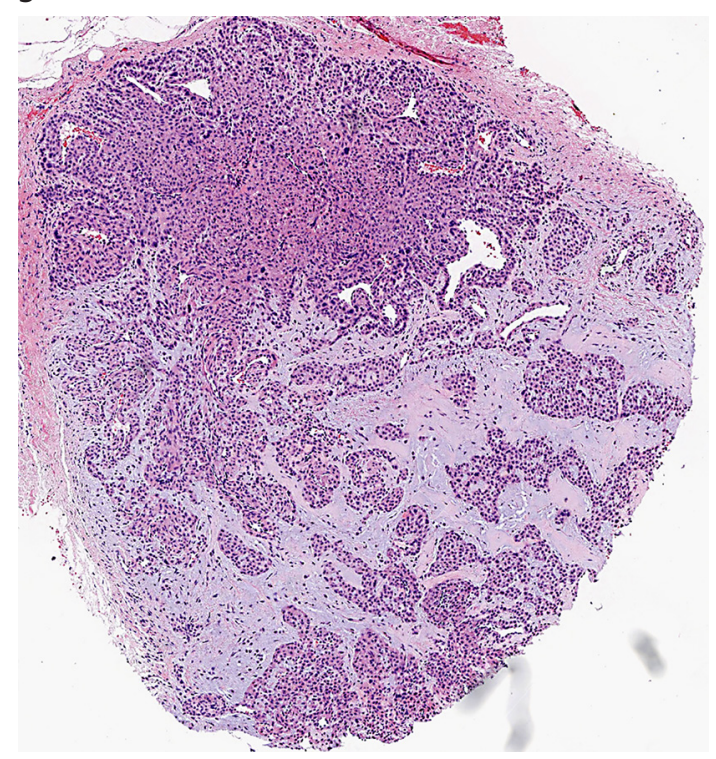

Fig. 3. Medium-power view demonstrates features of a glomus tumor, including cuboidal cells associated with dilated vascular channels. A distinctive myxoid stroma is present. At this power, it is possible to identify some of the lesional cells that demonstrate symplastic nuclear change (hematoxylin and eosin, $\times 45$ ).

Fig. 4. The lesional cells are highlighted with smooth muscle actin, confirming the diagnosis of a glomus tumor (smooth muscle actin, $\times 45$ ).

Fig. 5. Phosphohistone H3-stained sections demonstrate low proliferative activity of the lesional cells (phosphohistone $\mathrm{H} 3, \times 45$ ).

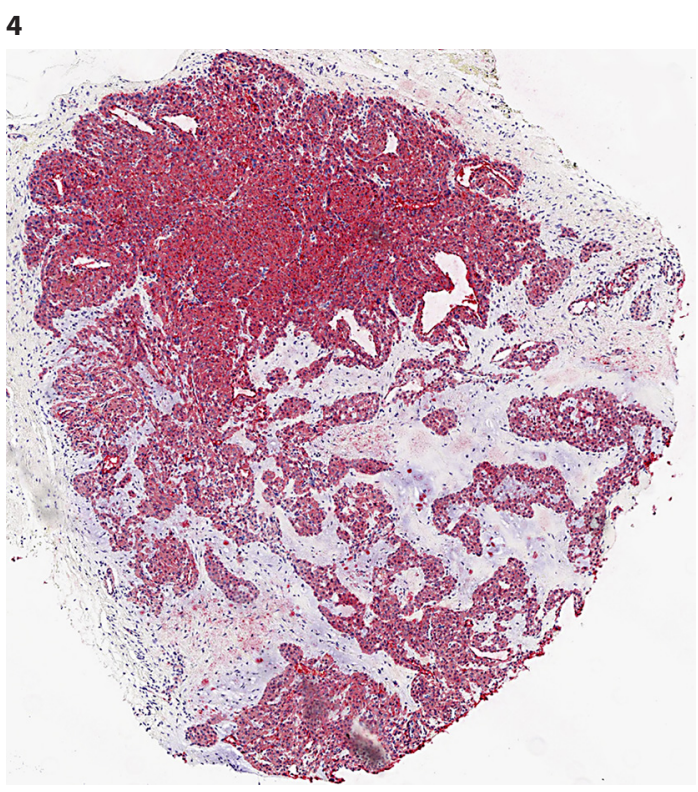

5

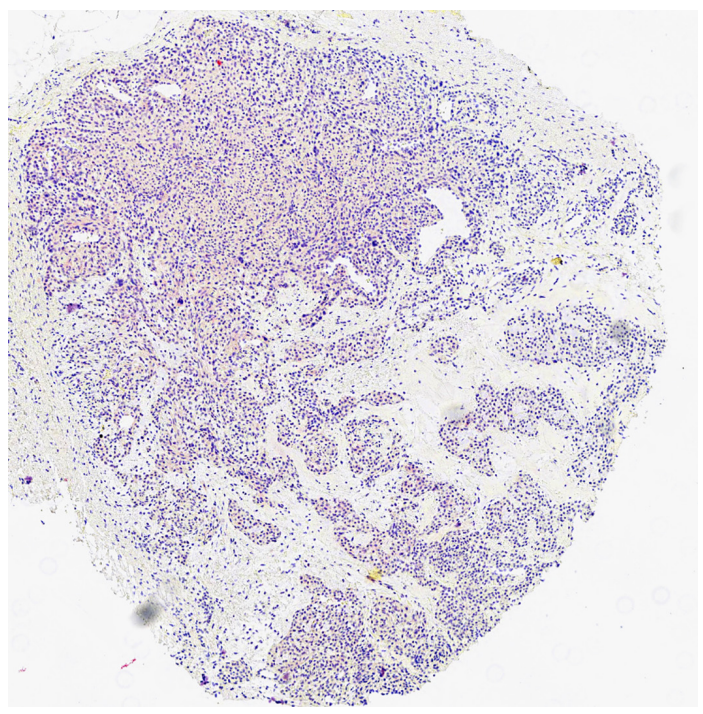

\section{Discussion}

Glomus tumors are unusual soft tissue tumors, which are most frequently encountered in the nail unit. Generally, these lesions are straightforward to diagnose by histopathology, but rare variants such as the variant described here, with myxoid and symplastic change, can cause diagnostic difficulty. The typical form of glomus tumor is a well-circumscribed lesion containing capillary-sized vasculature surrounded by glomus cells. A hyalinized or myxoid stroma within the parenchyma of a glomus tumor is an unusual feature. The monomorphous appearance of glomus cells, which are small polygonal cells with pale cytoplasm and typical round nuclei, is traditionally the most dependable clue to distinguishing this tumor from others with a comparable growth pattern [1,2]. Rarely, glomus tumors can display unusual histologic characteristics with significant nuclear atypia [1]. Folpe et al. [3] studied a series of 52 cases of glomus tumors with atypical features and designed a classification scheme which includes four groups. The malignant glomus tumor has a distinct risk of metastasis. These 
6

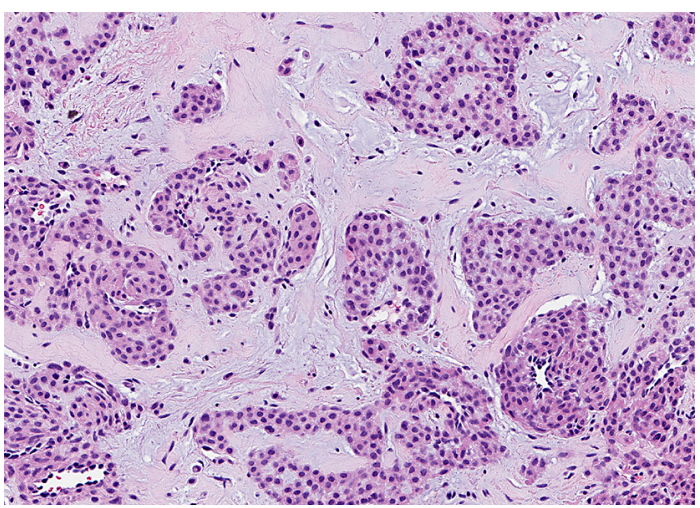

(c) 2018 The Author(s). Published by S. Karger AG, Basel www.karger.com/dpa

Da Silva et al.: Nail Unit Glomus Tumor with Myxoid and Symplastic Change

Fig. 6. The lesional cells are invested in a distinct myxoid stroma (hematoxylin and eosin, $\times 160$ ).

Fig. 7. Multiple glomus cells with symplastic change are seen, with enlarged and irregular nuclei and distinct nucleoli.

lesions fulfill at least one of the following criteria: increased depth and size more than $2 \mathrm{~cm}$, the presence of atypical mitotic figures, or a combination of moderate-to-high nuclear grade and mitotic activity ( 5 mitoses/50 high-power fields). Lesions exhibiting a combination of atypical features but which are not able to meet the minimum definition criteria of malignant glomus tumor are labeled "glomus tumors of uncertain malignant potential." The other group lacking criteria for malignant glomus tumor or glomus tumor of uncertain malignant potential is classified as "glomangiomatosis" and is characterized by diffuse infiltrative growth, resembling angiomatosis, with excess glomus cells. Lastly, glomus tumors having marked nuclear atypia in the absence of any other criteria for malignancy have been clearly shown to be benign and have been classified as "symplastic glomus tumor" [3].

In general, glomus tumors are most often subungual in location and commonly present as a small, slightly raised, violaceous or erythematous, painful nodule that can raise and distort the nail [4]. Localized tenderness and severe pain exacerbated by physical pressure or cold temperature are highly suggestive of this tumor. As with our case, glomus tumors may also present with localized longitudinal erythronychia, but this is a less common presentation [5-7]. In a Mexican study that examined all nail unit tumors in their health system, glomus tumor was 6th in frequency. Of their 13 cases, all demonstrated erythronychia and distal onycholysis but none displayed myxoid or symplastic change on histopathologic analysis [8]. Prominent myxoid change has been documented in glomus tumors $[9,10]$. This is also true of symplastic change, but there are only a few sources in the literature that demonstrate the combination of myxoid and symplastic change within a single glomus tumor specimen $[1,11]$. Kamarashev et al. [1] identified a lesion consistent with a symplastic glomus tumor with myxoid change, which presented with a pinpoint bluish discoloration of the nail associated with paroxysmal pain in the finger upon cold exposure. Mentzel et al. [11] also identified a lesion with both symplastic and myxoid change; however, it was not subungual in location.

It is worth noting that in an early case series, glomus tumors with histologic findings of symplastic glomus tumor were described as "epithelioid glomus tumors" in 1995 [2]. That series presented 5 cases, none of which were in the nail unit. This entity of epitheloid glomus tumor is histologically consistent with what is now referred to as symplastic glomus tumor.

Symplastic changes have been identified in several other tumors including giant cell tumors of bone, capillary arteriovenous malformations, and uterine leiomyomas. The common theme is that the nuclear atypia and pleomorphism mimic a malignant process but are decidedly benign in clinical course [12-14]. The high-grade nuclear atypia that characterizes 
these tumors is believed to be a manifestation of cellular degeneration and senescence rather than malignant transformation [1,3]. Importantly, symplastic glomus tumors are consistent with this and have not been shown to metastasize or recur after excision [3].

In summary, we present a case of a subungual glomus tumor with myxoid and symplastic change that had a clinical presentation of longitudinal erythronychia. This particular variant with both symplastic and myxoid change is rare and needs to be recognized as a benign entity by dermatopathologists to avoid the overdiagnosis of atypia and overly aggressive treatment. Of those glomus tumors with both myxoid and symplastic change, this tumor is the only one to have a clearly documented clinical presentation with longitudinal erythronychia. This clinical appearance is consistent with routine glomus tumors. Given the excellent clinicopathologic correlation of this case, we believe that glomus tumor with myxoid and symplastic change should be included to the clinical differential diagnosis of monodactylous longitudinal erythronychia.

\section{Statement of Ethics}

The manuscript was prepared in compliance with all ethical and confidentiality guidelines and principles. The patient gave her consent.

\section{Disclosure Statement}

The authors have no conflicts of interest to disclose.

\section{References}

1 Kamarashev J, French LE, Dummer R, Kerl K: Symplastic glomus tumor - a rare but distinct benign histological variant with analogy to other 'ancient' benign skin neoplasms. J Cutan Pathol 2009;36:1099-1102.

-2 Pulitzer DR, Martin PC, Reed RJ: Epithelioid glomus tumor. Hum Pathol 1995;26:1022-1027.

-3 Folpe AL, Fanburg-Smith JC, Miettinen M, Weiss SW: Atypical and malignant glomus tumors: analysis of 52 cases, with a proposal for the reclassification of glomus tumors. Am J Surg Pathol 2001;25:1-12.

4 Morey VM, Garg B, Kotwal PP: Glomus tumours of the hand: Review of literature. J Clin Orthop Trauma 2016; 7:286-291.

5 Jellinek NJ, Lipner SR: Longitudinal Erythronychia: Retrospective Single-Center Study Evaluating Differential Diagnosis and the Likelihood of Malignancy. Dermatol Surg 2016;42:310-319.

6 Micheletti R, Sobanko J, Rubin A: Distal matrix glomus tumor presenting as longitudinal erythronychia: a pearl for surgical management. Dermatol Surg 2012;38:133-134.

$>7$ Jellinek NJ: Longitudinal erythronychia: suggestions for evaluation and management. J Am Acad Dermatol 2011;64:167 e161-111.

-8 Dominguez-Cherit J, Chanussot-Deprez C, Maria-Sarti H, Fonte-Avalos V, Vega-Memije E, Luis-Montoya P: Nail unit tumors: a study of 234 patients in the dermatology department of the "Dr Manuel Gea Gonzalez" General Hospital in Mexico City. Dermatol Surg 2008;34:1363-1371.

-9 Hisa T, Nakagawa K, Wakasa K, Nagareda T, Hamada T: Solitary glomus tumour with mucinous degeneration. Clin Exp Dermatol 1994;19:227-229.

10 Godse KV: Glomus tumor with mucinous change. Indian J Dermatol Venereol Leprol 2005;71:367-368.

11 Mentzel T, Hugel H, Kutzner H: CD34-positive glomus tumor: clinicopathologic and immunohistochemical analysis of six cases with myxoid stromal changes. J Cutan Pathol 2002;29:421-425.

12 Sarungbam J, Agaram N, Hwang S, Lu C, Wang L, Healey J, Hameed M: Symplastic/pseudoanaplastic giant cell tumor of the bone. Skeletal Radiol 2016;45:929-935.

13 Samardzija G, Djuricic SM, Baljosevic I, Calonje E: Nasopharyngeal Capillary Arteriovenous Malformation with Ancient/Symplastic Change: A Simulator of Malignancy. Pediatr Dev Pathol 2016;19:249-253.

14 Downes KA, Hart WR: Bizarre leiomyomas of the uterus: a comprehensive pathologic study of 24 cases with long-term follow-up. Am J Surg Pathol 1997;21:1261-1270. 